\title{
PRECONDITIONERS FOR NONCONFORMING DISCRETIZATIONS
}

\author{
PETER OSWALD
}

\begin{abstract}
We prove an abstract norm equivalence for a two-level method, which allows us to reduce the construction of preconditioners for nonconforming finite element discretizations to known cases of conforming elements.
\end{abstract}

\section{INTRODUCTION}

The construction of fast solvers for nonconforming finite element discretizations has attracted the attention of many researchers. In the context of multilevel or multigrid-like methods, the main difficulty is the fact that the natural sequences of nonconforming finite element subspaces are nonnested and form external approximations involving modified bilinear forms and norms. In comparison to the variational multilevel theory for conforming finite element schemes (cf. [47, 49, 7, 38] for some surveys), one has to be careful with designing the so-called intergrid transfer operators (restriction and prolongation) and to control all other perturbations.

Attempts to propose a general theory in this direction have been made several times, see e.g. the papers by Bramble, Pasciak, Xu [8], Dörfler [26, 27]. We also mention a series of contributions by Brenner [10, 11, 12, 13, 14], where the most popular simple nonconforming elements have been examined. Many papers $[6,10,48,35,36,45]$ have been written on the nonconforming P1-element originally introduced by Crouzeix, Raviart [22]. For this particular element, the analysis may be considerably simplified by observing that the conforming linear element functions form a proper subspace of the set of nonconforming P1-elements on the same triangulation, see $[48,36,45]$. Roughly speaking, there is a very simple twolevel method reducing the nonconforming discretization to the conforming one for which fast solvers are already available.

In this paper we want to generalize this idea. In $\S 2$ we give an abstract theory for a two-level method, which allows to switch from a given symmetric positive definite variational problem

$\widetilde{(\mathrm{VP})} \quad$ Find $\tilde{u} \in \tilde{V}$ such that $\quad \tilde{a}(\tilde{u}, \tilde{v})=\tilde{\Phi}(\tilde{v}), \quad \forall \tilde{v} \in \tilde{V}$,

to another one,

$$
\text { Find } u \in V \text { such that } a(u, v)=\Phi(v), \quad \forall v \in V .
$$

The applications to nonconforming finite element discretizations for second- and fourth-order elliptic problems are given in $\S 3$. For a number of examples, we show

Received by the editor January 24, 1994 and, in revised form, February 11, 1995.

1991 Mathematics Subject Classification. Primary 65F10, 65F35, 65N22, 65N30.

Key words and phrases. Nonconforming finite elements, preconditioned iterative methods. 
how to verify the assumptions of the abstract theory. The main ingredients are auxiliary discrete scaled $L_{2}$ bilinear forms and appropriate quasi-interpolant operators $Q: \tilde{V} \rightarrow V$ resp. $\tilde{Q}: V \rightarrow \tilde{V}$ that preserve locally polynomials of low degree. After switching to (VP), multilevel preconditioning for the nonconforming discretization $(\widetilde{\mathrm{VP}})$ is a consequence of the known theory of multilevel preconditioners for conforming linear elements resp. Powell-Sabin triangles for secondresp. fourth-order problems. However, other types of preconditioners for (VP) can be used as well.

The idea of switching to a spectrally equivalent reference discretization is clearly not restricted to the cases considered in this paper and has implicitly and explicitly been explored by other authors, too. It also applies to certain complicated conforming elements and to the situation when the fine-grid triangulation is not produced by a standard refinement process, as it is typical for the multilevel solvers. After finishing the first draft of this paper we have learned about recent papers by Sarkis [43], Cowsar [21] and Brenner [15, 16, 17] that deal with similar questions in a domain decomposition framework. However, our approach is different in the technical details and emphasizes the idea of using multilevel preconditioners for some loworder conforming elements in a canonical way in the construction of preconditioners for more complicated situations, especially for nonconforming discretizations.

\section{The ABSTRACT NORM EQUiVALENCE}

Throughout this paper, let $\tilde{V}$ and $V$ be two finite-dimensional real Hilbert spaces. Let $\tilde{a}: \tilde{V} \times \tilde{V} \rightarrow \mathbf{R}$ resp. $a: V \times V \rightarrow \mathbf{R}$ be symmetric positive definite (spd) bilinear forms, and $\tilde{\Phi} \in \tilde{V}^{*}$ resp. $\Phi \in V^{*}$ be linear functionals on $\tilde{V}$ resp. $V$. We consider the variational problems $(\widetilde{\mathrm{VP}})$ and $(\mathrm{VP})$ as formulated in the introduction.

Assume that $W=\tilde{V}+V$ makes sense as a Hilbert space, and let there be given two further auxiliary spd bilinear forms $\hat{a}, b: W \times W \rightarrow \mathbf{R}$. In the applications below, $b$ represents an appropriately scaled $L_{2}$ scalar product while $\hat{a}$ is a suitable spectrally equivalent extension of both $\tilde{a}$ resp. $a$. Having this in mind, the following additional assumptions seem to be natural (here and in the sequel, generic constants are denoted by $c(0<c<\infty)$, and $\approx$ stands for a two-sided inequality):

(A1) The restrictions of $\hat{a}$ to $\tilde{V} \times \tilde{V}$ resp. $V \times V$ satisfy

$$
\tilde{a}(\tilde{v}, \tilde{v}) \approx \hat{a}(\tilde{v}, \tilde{v}), \quad a(v, v) \approx \hat{a}(v, v) \quad \forall \tilde{v} \in \tilde{V}, \quad \forall v \in V .
$$

(A2) The following inverse property holds:

$$
\hat{a}(w, w) \leq c \cdot b(w, w) \quad \forall w \in W .
$$

(A3) There exist linear operators $Q: \tilde{V} \rightarrow V$ and $\tilde{Q}: V \rightarrow \tilde{V}$ such that

$$
\begin{array}{ll}
b(\tilde{v}-Q \tilde{v}, \tilde{v}-Q \tilde{v}) \leq c \cdot \hat{a}(\tilde{v}, \tilde{v}) & \forall \tilde{v} \in \tilde{V}, \\
b(v-\tilde{Q} v, v-\tilde{Q} v) \leq c \cdot \hat{a}(v, v) & \forall v \in V .
\end{array}
$$

Theorem 1. With the above notations and the assumptions (A1), (A2), (A3), we have

$$
\begin{array}{ll}
\tilde{a}(\tilde{v}, \tilde{v}) \approx \inf _{\tilde{w} \in \tilde{V}, v \in V: \tilde{v}=\tilde{w}+\tilde{Q} v}\{b(\tilde{w}, \tilde{w})+a(v, v)\}, & \forall \tilde{v} \in \tilde{V}, \\
a(v, v) \approx \inf _{w \in V, \tilde{v} \in \tilde{V}: v=w+Q \tilde{v}}\{b(w, w)+\tilde{a}(\tilde{v}, \tilde{v})\}, & \forall v \in V,
\end{array}
$$


with constants depending only on the constants in (A1), (A2), (A3).

Proof. Because of symmetry of all assumptions and formulations, it suffices to establish (1). First observe that the linear operators $Q$ and $\tilde{Q}$ are bounded:

$$
\begin{array}{ll}
\hat{a}(\tilde{Q} v, \tilde{Q} v) \leq c \cdot a(v, v), & \forall v \in V, \\
\hat{a}(Q \tilde{v}, Q \tilde{v}) \leq c \cdot \tilde{a}(\tilde{v}, \tilde{v}), \quad \forall \tilde{v} \in \tilde{V} .
\end{array}
$$

Indeed, we can start with the obvious inequality

$$
\hat{a}(\tilde{Q} v, \tilde{Q} v) \leq 2 \cdot(\hat{a}(v-\tilde{Q} v, v-\tilde{Q} v)+\hat{a}(v, v)) .
$$

Here, the first term is estimated using (A2) and (A3) as follows :

$$
\hat{a}(v-\tilde{Q} v, v-\tilde{Q} v) \leq c \cdot b(v-\tilde{Q} v, v-\tilde{Q} v) \leq c \cdot \hat{a}(v, v) .
$$

Applying the second equivalence of (A1) gives (3); the proof of (4) is completely analogous.

Let $\tilde{v}=\tilde{w}+\tilde{Q} v$ be an arbitrary decomposition for any given $\tilde{v} \in \tilde{V}(\tilde{w} \in \tilde{V}, v \in$ $V)$. From the first equivalence in (A1), (A2), and (3), we get

$$
\tilde{a}(\tilde{v}, \tilde{v}) \leq c \cdot \hat{a}(\tilde{v}, \tilde{v}) \leq c \cdot(\hat{a}(\tilde{w}, \tilde{w})+\hat{a}(\tilde{Q} v, \tilde{Q} v)) \leq c \cdot(b(\tilde{w}, \tilde{w})+a(v, v)) .
$$

The upper bound in (1) follows by taking the infimum with respect to all decompositions.

To prove the opposite inequality, consider the particular decomposition of $\tilde{v} \in \tilde{V}$ given by

Since by $(\mathrm{A} 3)$

$$
v=Q \tilde{v}, \quad \tilde{w}=\tilde{v}-\tilde{Q} v=\tilde{v}-Q \tilde{v}+v-\tilde{Q} v .
$$

$$
b(\tilde{w}, \tilde{w}) \leq 2 \cdot(b(\tilde{v}-Q \tilde{v}, \tilde{v}-Q \tilde{v})+b(v-\tilde{Q} v, v-\tilde{Q} v)) \leq c \cdot(\hat{a}(\tilde{v}, \tilde{v})+\hat{a}(v, v)),
$$

and by (A1) and (4) also

$$
\hat{a}(\tilde{v}, \tilde{v}) \leq c \cdot \tilde{a}(\tilde{v}, \tilde{v}), \quad a(v, v) \leq c \cdot \hat{a}(v, v)=c \cdot \hat{a}(Q \tilde{v}, Q \tilde{v}) \leq c \cdot \tilde{a}(\tilde{v}, \tilde{v}),
$$

we get

$$
b(\tilde{w}, \tilde{w})+a(v, v) \leq c \cdot \tilde{a}(\tilde{v}, \tilde{v})
$$

for the above decomposition which shows the assertion.

As was stated in the introduction, the norm equivalence (1) is the basis for a certain two-level method to switch from $(\widetilde{\mathrm{VP}})$ to $(\mathrm{VP})$. This two-level method corresponds to the additive subspace splitting

$$
\tilde{V}=\tilde{V}+\tilde{Q} V,
$$

where the three spaces are equipped with appropriate bilinear forms such that (1) coincides with the basic norm equivalence for bounding the condition number of the corresponding additive Schwarz operator. Note that (2) might be used to switch from $(\mathrm{VP})$ back to $(\widetilde{\mathrm{VP}})$, a possibility we will not make use of in this paper.

To be more precise, let us recall the formulation of the so-called fictitious space lemma by Nepomnyaschikh $[33,34]$, see also $[38,29]$. Let $H_{0}$ and $H_{1}$ be two finitedimensional Hilbert spaces, with the scalar products denoted by $(\cdot, \cdot)_{0}$ resp. $(\cdot, \cdot)_{1}$. Let the bilinear forms $a_{0}: H_{0} \times H_{0} \rightarrow \mathbf{R}$ resp. $a_{1}: H_{1} \times H_{1} \rightarrow \mathbf{R}$ be generated by the spd operators $A_{0}: H_{0} \rightarrow H_{0}$ resp. $A_{1}: H_{1} \rightarrow H_{1}$. Finally, consider 
any surjective linear operator $R: H_{1} \rightarrow H_{0}$, together with its adjoint operator $R^{*}: H_{0} \rightarrow H_{1}$ given by $\left(R u_{1}, u_{0}\right)_{0}=\left(u_{1}, R^{*} u_{0}\right)_{1}$ for all $u_{1} \in H_{1}$ and $u_{0} \in H_{0}$.

Lemma 1. Under the above assumptions, we have the identity

$$
a_{0}\left(\left(R A_{1}^{-1} R^{*} A_{0}\right)^{-1} u_{0}, u_{0}\right)=\left.\left|\| u_{0}\right|\right|^{2} \equiv \inf _{v_{1} \in H_{1}: R v_{1}=u_{0}} a_{1}\left(v_{1}, v_{1}\right),
$$

for all $u_{0} \in H_{0}$. As a consequence, the minimal and maximal eigenvalues of the linear operator $R A_{1}^{-1} R^{*} A_{0}$ can be given by

$$
\lambda_{\min }=\inf _{u_{0} \in H_{0} \backslash\{0\}} \frac{a_{0}\left(u_{0}, u_{0}\right)}{\left\|\left|u_{0}\right|\right\|^{2}}, \quad \lambda_{\max }=\sup _{u_{0} \in H_{0} \backslash\{0\}} \frac{a_{0}\left(u_{0}, u_{0}\right)}{\left\|u_{0} \mid\right\|^{2}} .
$$

In the applications, it is assumed that $A_{1}$ can be easily inverted and that the multiplication by $R$ resp. its adjoint is also a cheap operation. Then, $R A_{1}^{-1} R^{*}$ can be used as preconditioner for $A_{0}$ provided that the ratio of the eigenvalues in (6) is not very large. The latter can be checked by evaluating the constants in the two-sided norm equivalence

$$
a_{0}\left(u_{0}, u_{0}\right) \approx\left\|u_{0}\right\|^{2} \quad, \quad \forall u_{0} \in H_{0} .
$$

To apply Lemma 1 to our situation, let us fix $H_{0}=\mathbf{R}^{\tilde{n}}$ and $H_{1}=\mathbf{R}^{\tilde{n}} \times \mathbf{R}^{n}$, where $\tilde{n}=\operatorname{dim} \tilde{V}, n=\operatorname{dim} V$. The connection between the vectors from $H_{0}$, $H_{1}$ and the finite element functions in $\tilde{V}, \tilde{V} \times V$, resp., will be provided by the standard nodal bases in $\tilde{V}$ and $V$. Denote by $\tilde{A}, \tilde{B}$ resp. $A$ the stiffness matrices of the bilinear forms $\tilde{a}, b$ (restricted to $\tilde{V} \times \tilde{V}$ ) resp. $a$ in these bases. Then we set

$$
a_{0}(\tilde{x}, \tilde{y})=(\tilde{A} \tilde{x}, \tilde{y})_{\mathbf{R}^{\tilde{n}}} \equiv \tilde{a}(\tilde{u}, \tilde{v})
$$

for all $\tilde{x}, \tilde{y} \in H_{0}$, where $\tilde{u}, \tilde{v} \in \tilde{V}$ are the finite element functions corresponding to $\tilde{x}, \tilde{y}$ via the nodal basis in $\tilde{V}$, and

$$
a_{1}((\tilde{x}, x),(\tilde{y}, y))=(\tilde{B} \tilde{x}, \tilde{y})_{\mathbf{R}^{\tilde{n}}}+(A x, y)_{\mathbf{R}^{n}} \equiv b(\tilde{u}, \tilde{v})+a(u, v)
$$

for arbitrary $(\tilde{x}, x),(\tilde{y}, y) \in H_{1}$ (in analogy, $u, v \in V$ are the functions associated with $x, y \in \mathbf{R}^{n}$ through the nodal basis in $V$ ). Finally, denote by $\tilde{S}$ the $n \times \tilde{n}$ matrix describing the action of $\tilde{Q}$ in the respective nodal basis representations, and set

$$
R(\tilde{x}, x)=\tilde{x}+\tilde{S} x
$$

which is obviously a mapping from $H_{1}$ onto $H_{0}$. With all this notation at hand, we see that (7) reduces exactly to the norm equivalence (1). Thus, the constants in the upper and lower estimate contained in (1) describe the quality of preconditioning the matrix $\tilde{A}$ by the symmetric preconditioner $\tilde{B}^{-1}+\tilde{S} A^{-1} \tilde{S}^{T}$. Moreover, $\tilde{B}^{-1}$ and $A^{-1}$ can be replaced by any symmetric preconditioner $\tilde{C}_{b}$ and $C_{a}$, resp. We subsume this in the following

Theorem 2. Under the above notations and the assumptions of Theorem 1, the spectral condition number of the matrix $\left(\tilde{B}^{-1}+\tilde{S} A^{-1} \tilde{S}^{T}\right) \tilde{A}$ equals the ratio of the best constants in the upper and lower inequality of the norm equivalence (1). In addition, if $\tilde{C}_{b}$ and $C_{a}$ are symmetric preconditioners for $\tilde{B}$ and $A$, resp., and satisfy the spectral bounds

$$
\lambda_{\max }\left(\tilde{C}_{b} \tilde{B}\right), \lambda_{\max }\left(C_{a} A\right) \leq \Lambda_{\max }, \quad \lambda_{\min }\left(\tilde{C}_{b} \tilde{B}\right), \lambda_{\min }\left(C_{a} A\right) \geq \Lambda_{\min },
$$


then $\tilde{C}_{\tilde{a}}=\tilde{C}_{b}+\tilde{S} C_{a} \tilde{S}^{T}$ is a symmetric preconditioner for $\tilde{A}$ with a bound for the spectral condition number of $\tilde{C}_{\tilde{a}} \tilde{A}$ depending on the constants in (8) and (1):

$$
\kappa\left(\tilde{C}_{\tilde{a}} \tilde{A}\right) \leq \frac{\Lambda_{\max }}{\Lambda_{\min }} \kappa\left(\left(\tilde{B}^{-1}+\tilde{S} A^{-1} \tilde{S}^{T}\right) \tilde{A}\right) .
$$

In most of the applications, the auxiliary form $b$ will be a scaled $L_{2}$ scalar product which leads, after switching to its discrete relatives in the nodal basis representation of $\tilde{V}$, to a simple diagonal matrix for the preconditioner $\tilde{C}_{b}$. For $C_{a}$ (the preconditioner for the reference problem $(\widetilde{\mathrm{VP}})$ on $V$ ), we favor multilevel methods for which a simple, regularity-free theory is available, see [38]. This, however, has restricted our choices for the reference discretization (VP) in this paper. An alternative are symmetric multigrid preconditioners as analyzed, e.g., in [7], which are available in many more situations.

The complexity of $\tilde{S}$ resp. $\tilde{S}^{T}$ depends on the choice for the operator $\tilde{Q}$, more precisely, on its representation with respect to the nodal bases of $\tilde{V}$ and $V$. Note that the operator $Q$ does not play any role in the corresponding algorithm. Since, for the typical choices of the forms $\tilde{a}$, $a$ resp. $b\left(H^{s}\right.$ inherited resp. scaled $L_{2}$ scalar products), the condition (A3) is fulfilled if $\tilde{Q}$ and $Q$ provide good $L_{2}$ approximation for smooth functions, we use quasi-interpolant techniques for their construction. This automatically leads to sparse matrices $\tilde{S}$. Details will be explained in $§ 3.2$.

\section{PreConditioners For NONCONFORMing ELEMENTS}

3.1. The nonconforming P1 element. To highlight the main ideas, and to start with a simple example, let us consider the discretization of the Poisson equation equipped with zero boundary conditions by nonconforming triangular P1 elements in $\mathbf{R}^{2}$ (the three-dimensional case is fully analogous). Let $\mathcal{T}$ be any fixed regular but not necessarily quasi-uniform triangulation of a bounded polygonal domain $\Omega$ in the plane, and introduce $\tilde{V}$ as the set of all piecewise linear functions with respect to $\mathcal{T}$ which are continuous at the midpoints $M_{e}$ of all interior edges $e$ and take zero values at the midpoints of boundary edges. To each interior edge $e$ we associate the nodal basis function $N_{e} \in \tilde{V}$ which is 1 at $M_{e}$ and zero at all other midpoints. The form on $\tilde{V}$ is given by

$$
\tilde{a}(\tilde{u}, \tilde{v})=\sum_{\Delta \in \mathcal{T}} \int_{\Delta} \nabla \tilde{u} \cdot \nabla \tilde{v} d x
$$

As a standard candidate for the corresponding problem (VP), we take the space of conforming linear elements $V=\tilde{V} \cap H_{0}^{1}(\Omega)$ on the same triangulation, equipped with

$$
a(u, v)=\tilde{a}(u, v)=\int_{\Omega} \nabla u \cdot \nabla v d x
$$

The standard nodal basis functions in $V$ will be denoted by $N_{P}$, where $P$ is in the set of interior vertices of $\mathcal{T}$.

Since $V \subset \tilde{V}$ we have $W=\tilde{V}$. The auxiliary forms $\hat{a}, b$ can be given by the formulae

$$
\hat{a}(\tilde{u}, \tilde{v})=\tilde{a}(\tilde{u}, \tilde{v}), \quad b(\tilde{u}, \tilde{v})=\sum_{e} \tilde{u}\left(M_{e}\right) \cdot \tilde{v}\left(M_{e}\right), \quad \forall \tilde{u}, \tilde{v} \in \tilde{V}
$$

(summation with respect to all interior edges). If the triangulation is in addition quasi-uniform, with $h$ denoting the typical size of the triangles, then $b(\tilde{u}, \tilde{u}) \approx$ 
$h^{-2} \cdot\|\tilde{u}\|_{L_{2}}^{2}$, i.e., the form $b$ essentially represents a scaled $L_{2}$ scalar product in this case. Obviously, (A1) and (A2) are satisfied, the latter property following from

$$
\hat{a}(\tilde{u}, \tilde{u})=\tilde{a}(\tilde{u}, \tilde{u}) \approx \sum_{\Delta \in \mathcal{T}} \sum_{e, e^{\prime} \subset \bar{\Delta}}\left(\tilde{u}\left(M_{e}\right)-\tilde{u}\left(M_{e^{\prime}}\right)\right)^{2}
$$

(here, the regularity of the triangulation enters the constants).

To verify (A3), we need to define $Q$ and $\tilde{Q}$. For $\tilde{Q}$ we take the natural imbedding of $V$ into $\tilde{V}$. Since for this choice $\tilde{Q} v=v$ for all $v \in V$, the required approximation property is obvious. The corresponding matrix $\tilde{S}$ (the matrix representation of $\tilde{Q}$ with respect to the above nodal bases) is very sparse, it contains two entries $\frac{1}{2}$ per column. The operator $Q$ does not enter the algorithm; thus, any choice that satisfies the estimate in (A3) (with a good constant) is acceptable. For example, we may assign to any interior vertex $P$ one of its adjacent edges (denoted by $e_{P}$ ) and define the nodal value of $Q \tilde{u} \in V$ at $P$ by $(Q \tilde{u})(P)=\tilde{u}\left(M_{e_{P}}\right)$; for boundary vertices we set $(Q \tilde{u})(P)=0$. Alternatively, one could propose to take the interior nodal values of $Q \tilde{u}$ as the average of the nodal values $\tilde{u}\left(M_{e}\right)$ for all $e$ adjacent to interior points (see, e.g., $[37,45])$.

In order to estimate $b(\tilde{u}-Q \tilde{u}, \tilde{u}-Q \tilde{u})$, observe that by definition of $Q$

$$
\left|\tilde{u}\left(M_{e}\right)-Q \tilde{u}\left(M_{e}\right)\right| \leq \frac{1}{2}\left(\left|\tilde{u}\left(M_{e}\right)-\tilde{u}\left(M_{e^{\prime}}\right)\right|+\left|\tilde{u}\left(M_{e}\right)-\tilde{u}\left(M_{e^{\prime \prime}}\right)\right|\right),
$$

where the edges $e, e^{\prime}$ resp. $e, e^{\prime \prime}$ have a common endpoint. Since for the regular triangulation under consideration the number of edges adjacent to an arbitrary vertex does not exceed a fixed number, the above two terms can be estimated by a fixed finite number of terms $\left|\tilde{u}\left(M_{e_{1}}\right)-\tilde{u}\left(M_{e_{2}}\right)\right|$, where each pair $e_{1}, e_{2}$ belongs to one of the triangles in $\mathcal{T}$. Note that each such pair does not occur (for all different e) more than a fixed number of times. Taking the squares of the expressions and summing up, we obtain from the definition of $b$ and by (10)

$$
\begin{aligned}
& b(\tilde{u}-Q \tilde{u}, \tilde{u}-Q \tilde{u})=\sum_{e}\left(\tilde{u}\left(M_{e}\right)-Q \tilde{u}\left(M_{e}\right)\right)^{2} \\
& \quad \leq c \cdot \sum_{\Delta \in \mathcal{T}} \sum_{e, e^{\prime} \in \bar{\Delta}}\left(\tilde{u}\left(M_{e}\right)-\tilde{u}\left(M_{e^{\prime}}\right)\right)^{2} \leq c \cdot \hat{a}(\tilde{u}, \tilde{u}),
\end{aligned}
$$

which is the estimate required in (A3).

Thus, by Theorem 2 we arrive at a preconditioner for the nodal basis stiffness matrix $\tilde{A}$ of the nonconforming P1 discretization of the form $C_{\tilde{A}}=\tilde{I}+\tilde{S} C_{A} \tilde{S}^{T}$. Here, $\tilde{I}$ is the $\tilde{n} \times \tilde{n}$ identity matrix associated with our specific form $b, \tilde{S}$ describes the action of $\tilde{Q}$ as explained above, and $C_{A}$ is an appropriately scaled (compare condition (8)) preconditioner for the nodal basis stiffness matrix $A$ corresponding to the conforming linear finite element discretization on the same partition $\mathcal{T}$. In [36], the BPX multilevel preconditioner [9] was used and tested for this purpose. In this case, (8) is satisfied with moderate constants, under minimal restrictions on the solution and the dyadic refinement process. Thus, in this case (triangulations obtained by regular dyadic refinement), the preconditioner $C_{\tilde{A}}$ yields uniformly bounded condition numbers $\kappa\left(C_{\tilde{A}} \tilde{A}\right)$ for all refinement levels. Obviously, for this choice of $C_{A}$ and $\tilde{S}$, the operation count per preconditioning step is proportional to the discretization size $\tilde{n}$. Since the BPX preconditioner is equally well-suited for triangulations obtained by nested refinement (see, e.g., $[4,23,38]$ ), and the above 
construction does not require quasi-uniformity, we can also work with reasonable adaptivity concepts in the nonconforming $\mathrm{P} 1$ element case (compare the recent paper [31]). But note again that the theory allows us to use for $C_{A}$ any (multilevel, multigrid, or other) symmetric preconditioner corresponding to the conforming discretization (VP).

Let us finish this subsection with some remarks. The same arguments can be used for a nonconforming P1 discretization of a Poisson problem in the 3-dimensional case. Also, preconditioning matrices for other nonconforming or conforming discretizations of more general second-order $H^{1}$ or $H_{0}^{1}$-elliptic boundary value problems can be designed along the same lines. Finally, though natural, the above choice of $\tilde{Q}$ is by no means the only one which satisfies the assumption (A3). For example, one could have associated with any interior edge $e$ one of its endpoints (denoted by $P_{e}$ ) and introduced $\tilde{Q}$ by

$$
\tilde{Q} u\left(M_{e}\right)=u\left(P_{e}\right), \quad \forall u \in V ;
$$

for boundary edges these values are 0 as before. Obviously,

$$
b(u-\tilde{Q} u, u-\tilde{Q} u)=\frac{1}{4} \sum_{e}\left|u\left(P_{e}\right)-u\left(P_{e}^{\prime}\right)\right|^{2} \leq c \cdot a(u, u)=c \cdot \hat{a}(u, u), \quad \forall u \in V,
$$

where $P_{e}^{\prime}$ denotes the second endpoint of $e$. This choice would further simplify the multiplication by $\tilde{S}$ resp. $\tilde{S}^{T}$ in the preconditioning step. A natural approach to the construction of suitable $Q, \tilde{Q}$ will be discussed in the following subsection.

3.2. Sufficient conditions for (A3). For more complicated situations, especially for higher-order elliptic problems like the plate bending problem discussed below, one is interested in simple criteria for the choice of $\tilde{Q}$ and $Q$. In the applications to second- and fourth-order elliptic problems, scaled (discrete or continuous) $L_{2}$ scalar products are typically used for the form $b$ while $a$ resp. $\tilde{a}$ are equivalent to modified $H^{l}$-seminorms for $l=1,2$. In this case, the concept of quasi-interpolants will be useful. Suppose that $\tilde{V}$ and $V$ are finite-dimensional spaces of finite-element-like piecewise polynomials over a regular partition $\mathcal{T}$. To each subregion $\Delta \in \mathcal{T}$ we assign an enlarged set $K(\Delta)$ which is the union of $\Delta$ and possibly some of the $\Delta^{\prime}$ adjacent with $\Delta$ (adjacent means that the closures of $\Delta$ and $\Delta^{\prime}$ have common points). In most of the standard finite element examples, we can even assume that $K(\Delta)=\Delta$, but in some cases a larger $K(\Delta)$ is needed, see $\S 3.3$. By the regularity of $\mathcal{T}$, we have $h\left(\Delta^{\prime}\right) \approx h(\Delta)$ for the diameters of all $\Delta^{\prime} \in K(\Delta)$. Since the evaluation of $\tilde{Q} u$ enters the algorithm and we are interested in a maximally local and simple construction, the following restricted definition of quasi-interpolants will be used; an analogous definition applies to $Q$. We call $\tilde{Q}: V \rightarrow \tilde{V}$ quasi-interpolant of order $l \geq 1$ if

- it is local and $L_{2}$ bounded, i.e.,

$$
\tilde{Q} u=0 \quad \text { on } \Delta, \quad \forall u \in V: u=0 \quad \text { on } K(\Delta),
$$

and

$$
\|\tilde{Q} u\|_{L_{2}(\Delta)} \leq c \cdot\|u\|_{L_{2}(K(\Delta))}
$$

with a constant $c$ independent of $\Delta \in \mathcal{T}$ and $u \in V$, and

- preserves polynomials of total degree $<l$, i.e.,

$$
\tilde{Q} p=p
$$


for all polynomials $p$ of degree $<l$ (it is assumed without further mentioning that the restrictions of all such polynomials to $\Omega$ belong to the natural domain of definition of $\tilde{Q})$.

Concerning the bilinear forms, we assume in addition to (A1) that

$$
b(\tilde{w}, \tilde{w}) \leq c \cdot \sum_{\Delta \in \mathcal{T}} h(\Delta)^{-2 l} \cdot \alpha(\Delta) \cdot\|\tilde{w}\|_{L_{2}(\Delta)}^{2}, \quad \forall \tilde{w} \in W,
$$

and

$$
\sum_{\Delta \in \mathcal{T}} \alpha(\Delta) \cdot|\tilde{w}|_{l, \Delta}^{2} \leq c \cdot \hat{a}(\tilde{w}, \tilde{w}), \quad \forall \tilde{w} \in W,
$$

for a certain set of moderately changing positive constants $\alpha(\Delta)$, i.e., we assume that the ratios $\alpha(\Delta) / \alpha\left(\Delta^{\prime}\right)$ are uniformly bounded for neighboring subregions $\Delta, \Delta^{\prime}$. These constants are typically determined by the forms $\tilde{a}$ resp. $a$ and can be used to model coefficient dependencies. Afterwards, $b$ is constructed such that (14) and (A2) hold true (the constants $\alpha(\Delta)$ will enter the diagonal preconditioner $\tilde{C}_{b}$ in a natural way). In (15), $|u|_{l, G}$ denotes the $H^{l}(G)$ seminorm controlling all lth-order derivatives of $u$.

One may generalize (14), (15) to a form which also includes lower-order local Sobolev seminorms in (15). This generalization is interesting for adequately dealing with lower-order Helmholtz terms but will not be further elaborated in this paper. Moreover, in all examples considered in the following subsections, we will take $\alpha(\Delta)=1$.

Lemma 2. Assume that $\tilde{Q}: V \rightarrow \tilde{V}$ and $Q: \tilde{V} \rightarrow V$ are quasi-interpolants of order $l$, and that the forms $\hat{a}, b$ satisfy (14), (15) with the same l. Finally, for any $\tilde{w} \in V \cup \tilde{V}$ and any $\Delta \in \mathcal{T}$ we assume a local Bramble-Hilbert estimate of the form

$$
\inf _{p: \operatorname{deg}(p)<l}\|\tilde{w}-p\|_{L_{2}(K(\Delta))}^{2} \leq c \cdot h(\Delta)^{2 l} \sum_{\Delta^{\prime} \subset K(\Delta)}|\tilde{w}|_{l, \Delta^{\prime}}^{2} .
$$

Then (A3) holds.

Proof. Consider the approximation property in (A3) for $\tilde{Q}$. By (14), (15), the problem reduces to local estimates of the following form: For any $\Delta \in \mathcal{T}$ and any polynomial $p$ of degree $<l$, using (11)-(13), we have

$$
\|v-\tilde{Q} v\|_{L_{2}(\Delta)} \leq\|v-p\|_{L_{2}(\Delta)}+\|\tilde{Q}(v-p)\|_{L_{2}(\Delta)} \leq c \cdot\|v-p\|_{L_{2}(K(\Delta))} .
$$

Taking the infimum with respect to $p$ and applying (16) to each $K(\Delta)$, we arrive at

$$
\|v-\tilde{Q} v\|_{L_{2}(\Delta)}^{2} \leq c \cdot h(\Delta)^{2 l} \cdot \sum_{\Delta^{\prime} \subset K(\Delta)}|v|_{l, \Delta^{\prime}}^{2} .
$$

To get $b(v-\tilde{Q} v, v-\tilde{Q} v) \leq c \cdot a(v, v)$, it remains to multiply by $\alpha(\Delta) \cdot h(\Delta)^{-2 l}$, to take into account the moderate change of $\alpha\left(\Delta^{\prime}\right)$ over $K(\Delta)$ and to look at (14), (15). The proof for the second estimate is completely analogous.

Although Lemma 2 looks very technical, most of its assumptions are easily verified. The condition (16) is necessary if $K(\Delta) \neq \Delta$ since $\tilde{V} \subset W$ is usually not contained in $H^{l}(\Omega)$ for nonconforming discretizations.

One has to be careful with extending the definition of $Q, \tilde{Q}$ such that (13) holds for all polynomials, especially near the boundary where boundary conditions may 


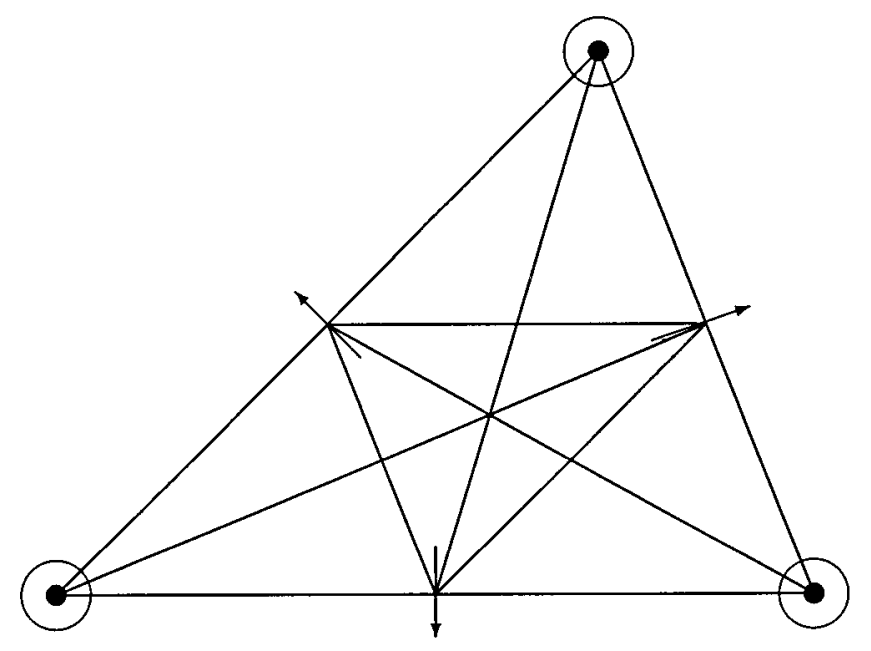

Figure 1. Powell-Sabin triangle

cause some technical problems. For example, in the definition of $Q$ for P1 elements given in $\S 3.1$, one would have to change the rule for the boundary vertices in order to have (12): For a vertex $P \in \partial \Omega$, there exists a boundary edge $e$ with $P$ as endpoint, and we define $Q \tilde{v}(P)=\tilde{v}\left(M_{e}\right)$ as for interior vertices. This modification does not change the $Q \tilde{u}$ for any $\tilde{u} \in \tilde{V}$, but now (13) is naturally satisfied (with $l=1$, i.e., constants are preserved). We hope that the following examples show that the quasi-interpolant concept is useful, and justify the separate formulation of Lemma 2.

3.3. The Morley triangle. The Morley triangle is of some importance in connection with the lowest-order divergence-free Raviart-Thomas element for the Stokes problem in two dimensions and also for plate bending. We refer to [18, 28] for generalities, and mention the papers [40, 26, 11,30], where multigrid methods and preconditioners are proposed. In contrast to these papers, we will relate the discretization of an appropriate fourth-order elliptic boundary value problem by Morley triangles to the corresponding conforming discretization by Powell-Sabin elements. Multilevel preconditioners with optimal convergence properties for the latter have been derived in [37].

We recall the definitions. We consider a clamped Kirchhoff plate which is modelled by the symmetric, $H_{0}^{2}$ elliptic, variational problem

$$
a(u, v) \equiv \int_{\Omega}\left\{\mu \sum_{i, j=1}^{2} \partial_{i j} u \partial_{i j} v+\lambda \Delta u \cdot \Delta v\right\} d x=\Phi(v) \equiv \int_{\Omega} f \cdot v d x
$$

with the solution $u \in H_{0}^{2}(\Omega)$ representing the deflection of the plate. The material constants $\mu, \lambda$ are fixed positive numbers.

Let $\mathcal{T}$ be as in $\S 3.1$; the finite element spaces $V$ resp. $\tilde{V}$ of conforming PowellSabin macrotriangles resp. nonconforming Morley triangles are composed from the local interpolation problems shown in Figures 1 and 2. The Powell-Sabin macrotriangle is a composite $C^{1}$ element consisting of quadratic polynomials in all 12 subtriangles which are uniquely determined by the following 12 degrees of freedom 


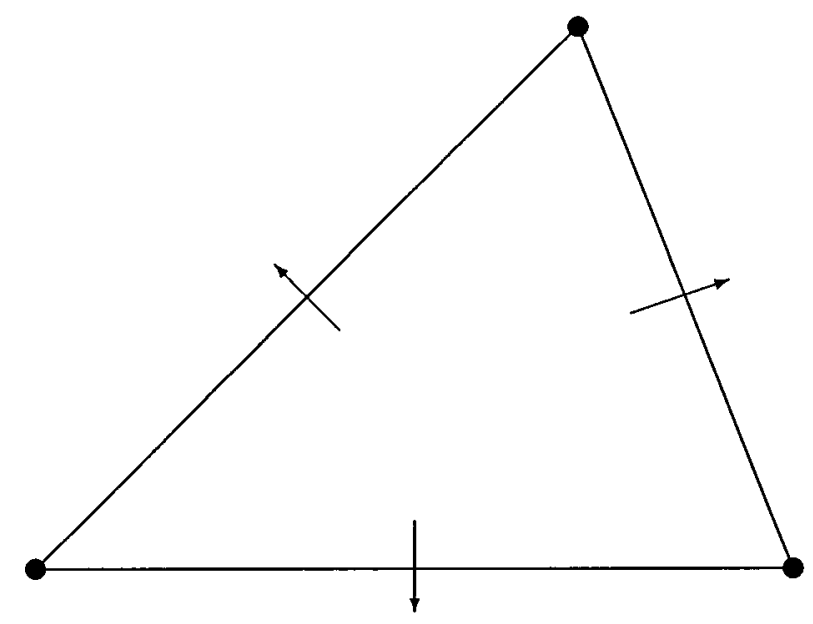

Figure 2. Morley triangle

(and the interior $C^{1}$ smoothness requirement): function values and gradients at the vertices and normal derivatives at the midpoints of the edges of the macrotriangle. At boundary vertices and edges, the degrees of freedom are set to zero to match the homogeneous boundary conditions. The resulting space is conforming $V \subset H_{0}^{2}(\Omega)$. The problem (VP) is defined simply as the restriction of (17) to $V$. Note that the Powell-Sabin element is, for several reasons, more popular for representing $C^{1}$ surfaces in computer aided geometric design than for the finite element analysis of plates. However, here it will not be used for the discretization itself but play the role of the reference space to construct a preconditioner for another discretization of (17).

In the case of the Morley element, in each triangle a quadratic polynomial is defined by the following 6 degrees of freedom: function values at the vertices and normal derivatives at the midpoints of the edges. Once again, to nodal points at the boundary we assign zero values. Since the resulting space is nonconforming $\left(\tilde{V} \not \subset H_{0}^{2}(\Omega)\right)$, the bilinear form on $\tilde{V}$ is usually changed (cf. [19]) into

$$
\tilde{a}(\tilde{u}, \tilde{v})=\sum_{\Delta \in \mathcal{T}} a\left(\left.\tilde{u}\right|_{\Delta},\left.\tilde{v}\right|_{\Delta}\right) \quad(\tilde{\Phi}(\tilde{v})=\Phi(\tilde{v})) .
$$

On $W$ we define the two auxiliary forms

$$
b\left(\tilde{w}, \tilde{w}^{\prime}\right)=\sum_{\Delta \in \mathcal{T}} h(\Delta)^{-4} \int_{\Delta} \tilde{w} \cdot \tilde{w}^{\prime} d x
$$

and

$$
\hat{a}\left(\tilde{w}, \tilde{w}^{\prime}\right)=\sum_{\Delta \in \mathcal{T}} \int_{\Delta} \sum_{i, j=1}^{2} \partial_{i j} \tilde{w} \partial_{i j} \tilde{w}^{\prime} d x .
$$

With this choice, the properties (A1), (14), and (15), here with $l=2$ and $\alpha(\Delta)=1$, are obvious, the constants may depend on $\mu, \lambda$, and on the regularity of $\mathcal{T}$. The inverse property (A2) reduces to local estimates on each triangle as usual, and is left to the reader. 
We introduce the quasi-interpolants $\tilde{Q}$ and $Q$. Let $\tilde{N}_{P}$ and $\tilde{N}_{e}$ denote the nodal basis functions for the Morley element case, and define

$$
\tilde{Q} v=\sum_{e}^{\prime} \partial_{n} v\left(M_{e}\right) \cdot \tilde{N}_{e}+\sum_{P}^{\prime} v(P) \cdot \tilde{N}_{P}, \quad \forall v \in V,
$$

where $\sum^{\prime}$ indicates that the summation is carried out for all (interior and boundary) edges resp. vertices. Clearly, $\tilde{Q}: V \rightarrow \tilde{V}$. This operator naturally extends onto $W$, and is the identity when restricted to $\tilde{V}$. It makes also sense for all quadratic polynomials and preserves them globally on $\Omega$. Thus, (13) is satisfied, even with $l=3$. The property (11) is obvious by definition, with the minimal choice $K(\Delta)=\Delta$, while (12) is a consequence of the local $L_{2}$ stability of both Morley and Powell-Sabin elements (which holds uniformly in $\Delta$ for regular triangulations):

$$
\begin{aligned}
& \|\tilde{Q} v\|_{L_{2}(\Delta)}^{2} \approx \sum_{e \subset \bar{\Delta}} h(\Delta)^{4} \partial_{n} \tilde{Q} v\left(M_{e}\right)^{2}+\sum_{P \in \bar{\Delta}} h(\Delta)^{2} \tilde{Q} v(P)^{2} \\
& \leq\left\{\sum_{e \subset \bar{\Delta}} h(\Delta)^{4} \partial_{n} v\left(M_{e}\right)^{2}+\sum_{P \in \bar{\Delta}}\left(h(\Delta)^{2} v(P)^{2}+h(\Delta)^{4}|\nabla v(P)|^{2}\right\} \approx\|v\|_{L_{2}(\Delta)}^{2} .\right.
\end{aligned}
$$

The operator $Q: \tilde{V} \rightarrow V$ is defined by

$$
\partial_{n} Q \tilde{v}\left(M_{e}\right)=\partial_{n} \tilde{v}\left(M_{e}\right), \quad Q \tilde{v}(P)=\tilde{v}(P),
$$

while the gradient values at a vertex $P$ are determined from two arbitrarily fixed non-collinear edges (for boundary vertices, these edges have to be at the boundary, too) such that globally linear polynomials are preserved. In the exceptional case where two boundary edges meet at an artificial vertex (with interior angle $\pi$ ), the construction of $Q$ has to be modified appropriately, i.e., the tangential derivative at the boundary vertex $P$ will be defined by the divided difference of function values of neighboring boundary vertices). Analogous modifications which ensure polynomial preservation with $l=2$ in (13) and zero boundary conditions of $Q \tilde{v}$ for all $\tilde{v} \in \tilde{V}$, are necessary if a slit has to be dealt with. Then, (11)-(13) can be verified for $Q$ in the same way as above. However, this time, $K(\Delta)$ should contain all $\Delta^{\prime}$ adjacent to $\Delta$; compare the definition of the gradient values of $Q \tilde{v}$ at vertices and the conditions (11)-(12).

Therefore, the verification of (16) is necessary for $\tilde{v} \in \tilde{V}$ (for $V \subset H^{2}(\Omega)$ the usual Bramble-Hilbert lemma will do the job). Let $p_{\Delta}$ be the quadratic polynomial coinciding with $\tilde{v}$ on $\Delta$, and let $\Delta^{\prime} \subset K(\Delta)$ be such that it shares the common edge $e$ with $\Delta$. Denote the endpoints of this $e$ by $P$ and $P^{\prime}$. The polynomial $q=p_{\Delta^{\prime}}-p_{\Delta}$ coincides with $\tilde{v}-p_{\Delta}$ on $\Delta^{\prime}$, and owing to the interpolation conditions for the Morley elements, we have $q(P)=q\left(P^{\prime}\right)=\partial_{n} q\left(M_{e}\right)=0$. Under this condition, we can easily check that

$$
\|q\|_{L_{2}\left(\Delta^{\prime}\right)} \leq c \cdot h(\Delta)^{2} \cdot|q|_{2, \Delta^{\prime}} .
$$

Indeed, without loss of generality, we may assume that $e=[-1,1]$ and that $h(\Delta) \approx$ $h\left(\Delta^{\prime}\right) \approx 1$. From $q(1)=q(-1)=\partial_{2} q(0)=0$ we see that $q\left(x_{1}, x_{2}\right)=\alpha\left(x_{1}^{2}-1\right)+$ $\beta x_{1} x_{2}+\gamma x_{2}^{2}$ while the second order partial derivatives of $q$ take constant values $\partial_{11} q\left(x_{1}, x_{2}\right)=2 \alpha, \partial_{12} q\left(x_{1}, x_{2}\right)=\beta, \partial_{22} q\left(x_{1}, x_{2}\right)=2 \gamma$ on $\mathbf{R}^{2}$, which gives the desired estimate. Since $q=\tilde{v}-p_{\Delta}=p_{\Delta^{\prime}}-p_{\Delta}$ on $\Delta^{\prime}$, we get

$$
h(\Delta)^{-2}\left\|\tilde{v}-p_{\Delta}\right\|_{L_{2}\left(\Delta^{\prime}\right)} \leq c|q|_{2, \Delta^{\prime}} \leq c\left\{\left|p_{\Delta}\right|_{2, \Delta^{\prime}}+\left|p_{\Delta^{\prime}}\right|_{2, \Delta^{\prime}}\right\} \leq c\left\{|\tilde{v}|_{2, \Delta}+|\tilde{v}|_{2, \Delta^{\prime}}\right\}
$$


for the three triangles $\Delta^{\prime} \subset K(\Delta)$ which possess a common edge with $\Delta$. Applying this estimation technique repeatedly, after finitely many steps we arrive at

$$
h(\Delta)^{-4} \cdot\left\|\tilde{v}-p_{\Delta}\right\|_{L_{2}(K(\Delta))}^{2} \leq c \cdot \sum_{\Delta^{\prime} \in K(\Delta)}|\tilde{v}|_{2, \Delta^{\prime}}^{2} .
$$

Now it remains to observe that $\left|p_{\Delta}\right|_{2, K(\Delta)} \approx|\tilde{v}|_{2, \Delta}$ and to approximate the quadratic polynomial $p_{\Delta}$ by arbitrary linear polynomials on $K(\Delta)$. This eventually gives (16) for $l=2$.

After this examination of all conditions of Lemma 2 and Theorem 1, we can state the main result of this subsection. We recall the notation used in Theorem 2 of $\S 2$. Let $\tilde{A}, \tilde{B}$ resp. $A$ be the nodal basis stiffness matrices of the forms $\tilde{a}, b$ on $\tilde{V}$ resp. $a$ on $V$. The matrix representation $\tilde{S}$ of $\tilde{Q}$ can be derived from (21), the multiplication by $\tilde{S}$ reduces to copying some entries of a vector from $\mathbf{R}^{n}$ into a shorter vector from $\mathbf{R}^{\tilde{n}}$ (in the present case, $\tilde{n}=\operatorname{dim} \tilde{V}<n=\operatorname{dim} V$ ). Since

$$
b(\tilde{v}, \tilde{v}) \approx \sum_{e} \partial_{n} \tilde{v}\left(M_{e}\right)^{2}+\sum_{P} h(P)^{-2} \cdot \tilde{v}(P)^{2}, \quad \forall \tilde{v} \in \tilde{V}
$$

where $h(P)$ is taken such that $h(P) \approx h(\Delta)$ for all $\Delta$ adjacent with the vertex $P$ and the summation is carried out for all interior edges $e$ resp. interior vertices $P$, $\tilde{B}$ possesses a diagonal preconditioner $\tilde{C}_{b}$ satisfying (8) with constants depending only on the regularity of $\mathcal{T}$. A multilevel preconditioner $C_{a}$ for the Powell-Sabin discretization matrix $A$, of optimal complexity and satisfying (8), is available from the theory in [37], if $\mathcal{T}=\mathcal{T}_{J}$ is obtained by $J$ steps of regular dyadic refinement from a coarse triangulation $\mathcal{T}_{0}$ of $\Omega$. Nested refinement has not been considered in [37]; an extension in this direction presents some technical but not principal difficulties (compare $\S 4.2 .2$ of [38]). For this reason we restrict the formulation of the following theorem to the regular refinement case.

Theorem 3. Let $\tilde{A}$ be the stiffness matrix of a nonconforming Morley finite element discretization of the plate bending problem (17) using the modified form (18) with respect to a triangulation $\mathcal{T}=\mathcal{T}_{J}$ obtained by regular dyadic refinement from $\mathcal{T}_{0}$. Then, uniformly in $J=1,2, \ldots$, we have

$$
\kappa\left(\left(\tilde{C}_{b}+\tilde{S} C_{a} \tilde{S}^{T}\right) \tilde{A}\right) \leq C,
$$

where the constant $C$ only depends on the regularity constant of the triangulation $\mathcal{T}_{0}$, and on $\mu$ and $\lambda$. The preconditioner $C_{\tilde{A}}=\tilde{C}_{b}+\tilde{S} C_{a} \tilde{S}^{T}$ requires $\mathrm{O}(\tilde{n})$ operations per matrix-vector multiplication and is based on a $V$-cycle multilevel preconditioner $C_{a}$ for the corresponding discretization by Powell-Sabin elements. The matrices $\tilde{S}$ and $\tilde{C}_{b}$ can be derived from (21)-(22) as explained above.

3.4. Zienkiewicz triangle. The interpolation conditions associated with the Zienkiewicz triangle (or reduced cubic Hermite triangle) are indicated in Figure 3. The local polynomial space is a 9-dimensional subspace of total degree-three polynomials $p$ satisfying the constraint

$$
6 p\left(S_{\Delta}\right)-\sum_{i=1}^{3}\left\{2 p\left(P_{i}\right)-\nabla p\left(P_{i}\right)\left(P_{i}-S_{\Delta}\right)\right\}=0,
$$

where $S_{\Delta}$ denotes the barycenter, and $P_{i}$ the vertices of the triangle $\Delta$. For any regular $\mathcal{T}$, the resulting finite element space $\tilde{V}$ is nonconforming with respect to 


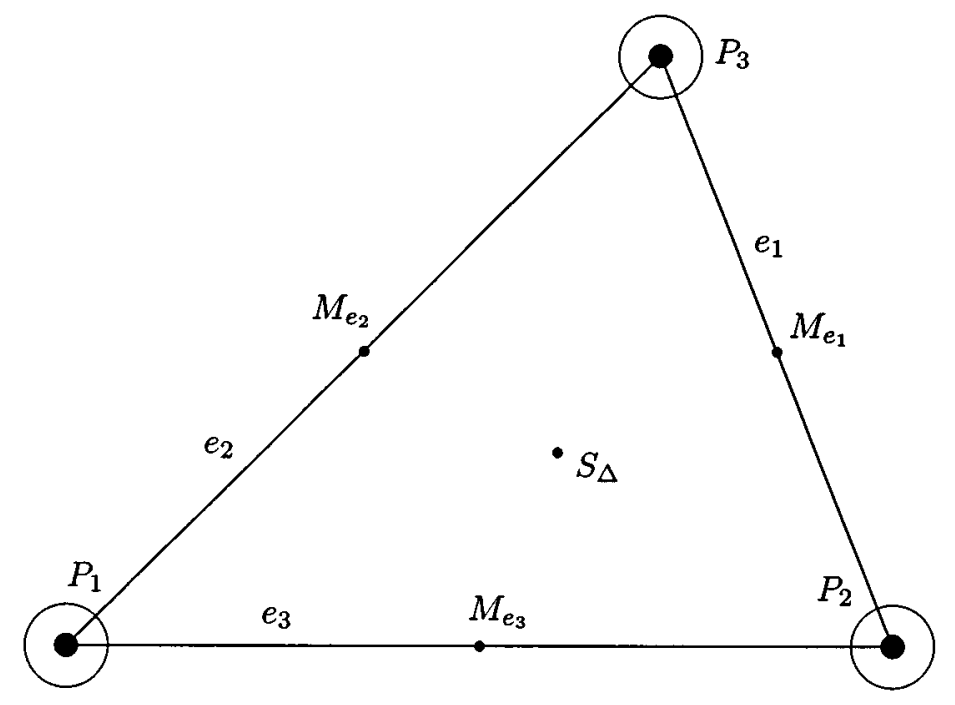

FiguRE 3. Reduced cubic (Zienkiewicz) triangle

$H_{0}^{2}(\Omega)$, the functions are $C^{0}$ but not $C^{1}$ (across interior edges). As before, boundary interpolation conditions are set to be zero.

There are different possibilities to introduce the modified form $\tilde{a}$ which replaces $a$ (given by (17)) on $\tilde{V}$. We quote the following three possibilities from [5, Ch. VI, $5]$, where references to the original papers are given (cf. also [19, 32]):

$$
\begin{gathered}
\tilde{a}_{0}(\tilde{u}, \tilde{v})=\sum_{\Delta \in \mathcal{T}} a\left(\left.\tilde{u}\right|_{\Delta},\left.\tilde{v}\right|_{\Delta}\right), \\
\tilde{a}_{1}(\tilde{v}, \tilde{v})=a^{*}(\theta \tilde{u}, \theta \tilde{v}), \\
\tilde{a}_{2}(\tilde{v}, \tilde{v})=a^{*}(\hat{\theta} \tilde{u}, \hat{\theta} \tilde{v})+\nu \sum_{\Delta \in \mathcal{T}} h(\Delta)^{-2} \int_{\Delta}(\nabla \tilde{u}-\hat{\theta} \tilde{u}) \cdot(\nabla \tilde{v}-\hat{\theta} \tilde{v}) d x
\end{gathered}
$$

for all $\tilde{u}, \tilde{v} \in \tilde{V}$. Here, $a^{*}(\phi, \psi)$ is a bilinear form defined for vector functions $\phi, \psi \in\left(H_{0}^{1}(\Omega)\right)^{2}$ such that $a(u, v)=a^{*}(\nabla u, \nabla v)$, i.e.,

$$
a^{*}(\phi, \psi)=\int_{\Omega} \mu \sum_{i, j=1}^{2} \epsilon_{i j}(\phi) \epsilon_{i j}(\psi)+\lambda \operatorname{div} \phi \operatorname{div} \psi d x, \quad \epsilon_{i j}(\phi) \equiv \frac{1}{2}\left(\partial_{i} \phi_{j}+\partial_{j} \phi_{i}\right) .
$$

The mappings $\theta$ and $\hat{\theta}$ are discrete gradient operators on $\tilde{V}: \theta \tilde{v}$ is componentwise defined by quadratic $C^{0}$ Lagrange element functions over $\mathcal{T}$ (with the components modelling the partial derivatives of $\tilde{v}$ ), with the nodal values given by $\nabla \tilde{v}\left(P_{i}\right)$ for vertices; at the midpoints $P_{i j}$ of the edges the corresponding values are obtained by orthogonal transformation from the directional derivative $\partial_{e} \tilde{v}\left(P_{i j}\right)$ and from $\frac{1}{2}\left(\partial_{n} \tilde{v}\left(P_{i}\right)+\partial_{n} \tilde{v}\left(P_{j}\right)\right)$, which stands for the nonexisting $\partial_{n} \tilde{v}\left(P_{i j}\right)$. By definition, $\hat{\theta}$ maps into the space of linear finite element vector fields over $\mathcal{T}$ according to $\hat{\theta} \tilde{v}(P)=\nabla \tilde{v}(P)$ for all vertices. Finally, $\nu>0$ is a positive parameter which we recommend to choose according to $\nu \approx \mu$ (see below). 
The following lemma is more or less known from the literature (cf. [5] and the original references cited therein) and states the coercivity of $\tilde{a}$ on $\tilde{V}$, which is necessary for the correctness of the variational problem $(\widetilde{\mathrm{VP}})$.

Lemma 3. The forms (24)-(26) are spd on $\tilde{V}$ and satisfy

$$
\begin{gathered}
c \mu \cdot \hat{a}(\tilde{u}, \tilde{u}) \leq \tilde{a}_{0}(\tilde{u}, \tilde{u}) \leq c(\mu+\lambda) \cdot \hat{a}(\tilde{u}, \tilde{u}), \\
c \mu \cdot \hat{a}(\tilde{u}, \tilde{u}) \leq \tilde{a}_{1}(\tilde{u}, \tilde{u}) \leq c(\mu+\lambda) \cdot \hat{a}(\tilde{u}, \tilde{u}), \\
c \min (\mu, \nu) \cdot \hat{a}(\tilde{u}, \tilde{u}) \leq \tilde{a}_{2}(\tilde{u}, \tilde{u}) \leq c(\mu+\lambda+\nu) \cdot \hat{a}(\tilde{u}, \tilde{u})
\end{gathered}
$$

for all $\tilde{u} \in \tilde{V}$, where $\hat{a}(\tilde{u}, \tilde{u})$ is given by (20). The constants $c$ in the two-sided estimates only depend on the regularity constant of the underlying triangulation.

We will leave the elementary proof to the reader (for $\tilde{a}_{0}$ the above result is obvious by definition of $a$ and of the $H^{2}$ seminorm). Note that the choice $\nu \approx \mu$ would be natural; however, in the context of Reissner-Mindlin plate models, the additional term in (26) has prescribed meaning, and the parameter $\nu$ is proportional to the thickness of the plate. We will not go into details, but rather refer to [5] for a discussion of this point.

Note also that the convergence properties of the discretizations corresponding to the above three modified forms are quite different. For example, with $\tilde{a}_{0}$ convergence is observed only for special triangulations; the formulation with $\tilde{a}_{1}$ which appears in the context of the DKT-element family was analyzed in [42], see also [41] or [5, Ch. VI, 5]. However, by virtue of Lemma 3 , there is no difference for the three formulations with respect to the construction of a preconditioner via Theorems 1 and 2 .

To this end, we again make use of the Powell-Sabin finite element subspace $V$ (see the subsection for the Morley element) on the same triangulation. Introduce $\tilde{Q}: V \rightarrow \tilde{V}$ in a natural way by defining the nodal values

$$
\tilde{Q} v(P)=v(P), \quad \nabla \tilde{Q} v(P)=\nabla v(P),
$$

at all vertices $P$ of $\mathcal{T}$, and $Q: \tilde{V} \rightarrow V$ by

$$
Q \tilde{v}(P)=\tilde{v}(P), \quad \nabla Q \tilde{v}(P)=\nabla \tilde{v}(P), \quad \partial_{n} Q \tilde{v}\left(M_{e}\right)=\frac{1}{2}\left(\partial_{n} \tilde{v}(P)+\partial_{n} \tilde{v}\left(P^{\prime}\right)\right),
$$

for all vertices $P$ and edges $e$ (for the latter, $P, P^{\prime}$ denote the endpoints of $e$ in the above formulae). This definition of $Q$ and $\tilde{Q}$ obviously guarantees (11)-(13) with $l=2$ and $K(\Delta)=\Delta$ in both cases. For the auxiliary forms on $W$ we use the definitions in (19) and (20), which makes it easy to prove the remaining assumptions of Lemma 2 and Theorem 1.

Indeed, (14), (15) are automatically satisfied (with $\alpha(\Delta)=1$ ). (A1) is contained in Lemma 3 for $\tilde{v} \in \tilde{V}$, for $v \in V$, see $\S 3.3$. The inverse property (A2) reduces by affine transformation to analyzing the situation on a standard triangle as usual, and is left to the reader. Finally, since $K(\Delta)=\Delta$ and the restriction of $V \cup \tilde{V}$ to a triangle $\Delta$ belongs to $H^{2}(\Delta),(16)$ follows from the usual Bramble-Hilbert lemma and a scaling argument.

After completing the verification of the assumptions of Lemma 2 and Theorem 1 , we can formulate the complete analog to Theorem 3 . With $\tilde{A}, \tilde{B}, A$ denoting the corresponding stiffness matrices, $\tilde{S}$ now defined by (27), we make again use of the multilevel preconditioner $C_{a}$ for the Powell-Sabin discretization matrix (compare 
$\S 3.3)$ while $\tilde{C}_{b}$ is a diagonal matrix derived from discretizing $b$ via the obvious equivalence

$$
\tilde{b}(\tilde{v}, \tilde{v}) \approx \sum_{P}\left\{|\nabla \tilde{v}(P)|^{2}+h(P)^{-2} \cdot \tilde{v}(P)^{2}\right\}, \quad \forall \tilde{v} \in \tilde{V} .
$$

Theorem 4. Let $\tilde{A}$ be the stiffness matrix of a nonconforming Zienkiewicz element discretization of the plate bending problem (17) by using any of the modified bilinear forms (24)-(26), where the underlying triangulation $\mathcal{T}=\mathcal{T}_{J}$ is obtained by regular dyadic refinement from $\mathcal{T}_{0}$. Then, uniformly in $J=1,2, \ldots$, we have

$$
\kappa\left(\left(\tilde{C}_{b}+\tilde{S} C_{a} \tilde{S}^{T}\right) \tilde{A}\right) \leq C,
$$

where the constant $C$ depends only on the regularity constant of the triangulation $\mathcal{T}_{0}$, and on $\mu, \lambda$, and $\nu$ (cf. Lemma 3). The preconditioner $C_{\tilde{A}}=\tilde{C}_{b}+\tilde{S} C_{a} \tilde{S}^{T}$ requires $\mathrm{O}(\tilde{n})$ operations per matrix-vector multiplication and is based on a $V$-cycle multilevel preconditioner $C_{a}$ for the corresponding discretization by Powell-Sabin elements, while $\tilde{S}, \tilde{C}_{b}$ are defined via (27)-(28) as explained.

\subsection{Further remarks.}

Other element types. In $\S \S 3.1,3.3,3.4$, we have discussed a few triangular nonconforming elements for second- resp. fourth-order boundary value problems. In these cases, we have chosen conforming linear elements resp. Powell-Sabin elements as reference elements, particularly, because for them optimal multilevel preconditioners are known. Complicated conforming elements such as Argyris and Bell triangles or composite Clough-Tocher triangles, which do not allow for a straightforward construction of multilevel preconditioners, may be dealt with in the same conceptional way (for a slightly different approach, see [50, 17]). Generalizations of the Morley element to higher polynomial degree such as the semiloof or delinquent elements (cf. [44]) can be treated as well. We leave it up to the reader to consider more examples, e.g., from the list of (conforming and nonconforming) elements for thin plate bending which is contained in [32, Ch. 7], see also [19, 20,5].

If the original discretization uses rectangular resp. quadrilateral elements, then for $H^{1}$ problems bilinear elements (for rectangles) or the reduction to triangular linear elements (for quadrilaterals) are an advisable choice for the reference space $V$. What concerns plate bending problems, we have at least three candidates (conforming $C^{1}$ elements of low order) where optimal multilevel preconditioners are known:

- Composite cubic quadrilaterals [24] (see Figure 4, where the 16 interpolation conditions are described which uniquely define the $C^{1}$ piecewise cubic function inside each quadrilateral; in the finite element literature these elements are attributed to Sanders and Fraeijs De Veubeke, see [19, 32]);

- Bogner-Fox-Schmit bicubic rectangles [39, 50];

- Biquadratic $C^{1}$ tensor-product splines (the theory for this space of $C^{1}$ test functions, which is closely related to standard finite difference discretizations on a shifted grid, follows easily from [38]).

Though the considerations in $[24,37,39,50]$ are restricted to regular refinement, it is clear that the theory of multilevel preconditioners for these conforming discretizations carries over to nested refinement (cf. [23, 38] concerning this point). Thus, the two-level reduction to standard multilevel solvers as proposed in the present 


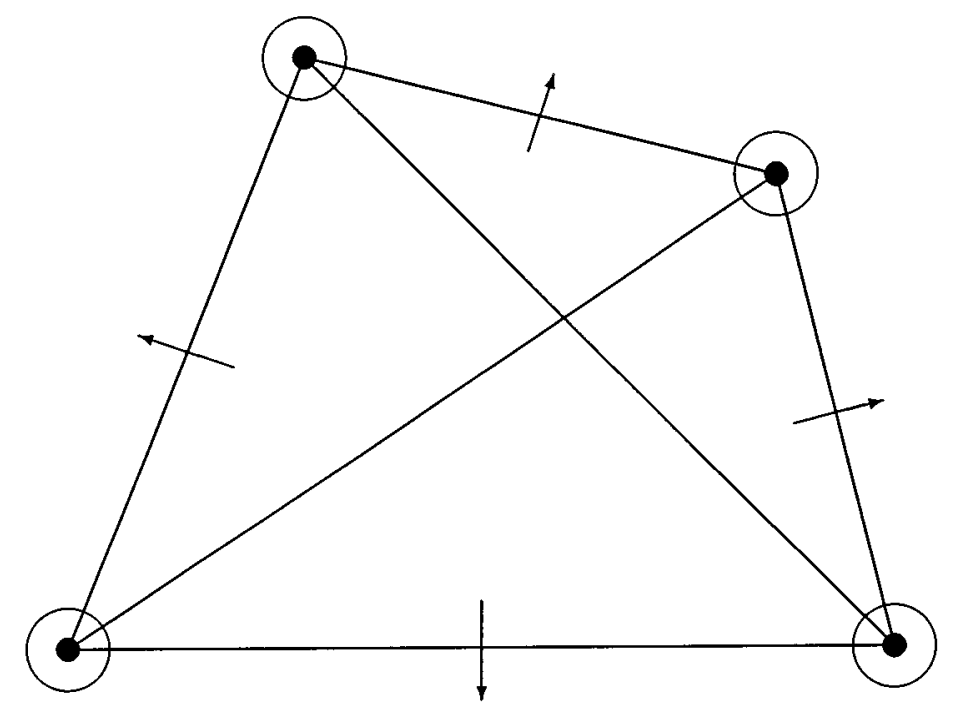

Figure 4. Composite cubic quadrilateral

paper will be possible for these element types, too. For example, the specific preconditioner for the nonconforming rectangular Adini element described in [39] is based on a similar (to the Bogner-Fox-Schmit element) reduction argument. For rectangular elements, the use of the biquadratic spline spaces for preconditioning purposes seems to be even more economic with respect to the operation count per preconditioning step.

The practical performance of our approach has still to be tested carefully. For example, an efficient implementation of the Powell-Sabin multilevel preconditioner [37], which we suggested to use in connection with triangular elements for plate bending (or of the composite cubic $C^{1}$ quadrilaterals considered in [24]) is, to our knowledge, not yet available. For the Bogner-Fox-Schmit and Adini elements, some experiments have been carried out in [39].

Construction of error estimators. The basic norm equivalence (1) might be useful for other purposes such as the construction of a posteriori error estimators. To give an example, let us denote by $\tilde{V} \equiv V_{Q}$ resp. $V \equiv V_{L}$ the subspaces of quadratic resp. linear finite element functions over a regular triangulation of a bounded polyhedral domain $\Omega$ in two dimensions (once again, consider the case of homogeneous Dirichlet conditions on the boundary). The three-dimensional case can be treated similarly. Let $a(\cdot, \cdot)$ be a symmetric $H_{0}^{1}$ elliptic variational problem which, when restricted to $V, \tilde{V}$, and $W=\tilde{V}$, leads to the bilinear forms used on these subspaces (to simplify the notation, we will use the letter $a$ also for $\tilde{a}$ and $\hat{a}$ ). Finally, introduce the auxiliary form $b$ on $\tilde{V}$ by

$$
b\left(\tilde{w}, \tilde{w}^{\prime}\right)=\sum_{\Delta} h(\Delta)^{-2} \int_{\Delta} \tilde{w} \cdot \tilde{w}^{\prime} d x .
$$

Then (1) holds true (we leave this to the reader as an exercise; since $V \subset \tilde{V}$ the operator $\tilde{Q}$ is the natural injection, while $Q$ corresponds to nodal interpolation by linear finite elements). 
Now, denote by $u_{Q}$ resp. $u_{L}$ the solutions of the variational problems ( $\left.\widetilde{\mathrm{VP}}\right)$ resp. (VP), where $\tilde{\Phi}, \Phi$ are restrictions of the same functional to the respective subspaces. Let $e=u_{Q}-u_{L}$, and define $\tilde{e}$ by

$$
b(\tilde{e}, \tilde{v})=a(e, \tilde{v}), \quad \forall \tilde{v} \in \tilde{V} .
$$

Then, the two-sided inequality

$$
a(e, \tilde{e})=b(\tilde{e}, \tilde{e}) \approx a(e, e)
$$

holds with the same constants as in (1). This is obvious if one uses the properties of the additive Schwarz operator corresponding to (1), see [47, 49, 29]. A direct argument runs as follows: Since $a(e, v)=0$ for all $v \in V$, we can write

$$
\begin{aligned}
& b(\tilde{e}, \tilde{e})^{2}=a(e, \tilde{e})^{2}=\inf _{v} a(e, \tilde{e}-v)^{2} \leq a(e, e) \cdot \inf _{v} a(\tilde{e}-v, \tilde{e}-v), \\
& a(e, e)^{2}=\inf _{v} a(e, e-v)^{2}=\inf _{v} b(\tilde{e}, e-v)^{2} \leq b(\tilde{e}, \tilde{e}) \cdot \inf _{v} b(e-v, e-v) .
\end{aligned}
$$

It remains to verify the identity

$$
\inf _{v} b(\tilde{u}-v, \tilde{u}-v)=\inf _{v}\|\tilde{u}-v\|^{2} \quad, \quad \forall \tilde{u} \in \tilde{V},
$$

where $\left.\|\tilde{v}\|\right|^{2}$ denotes the right-hand side in (1), and to apply this inequality in the appropriate directions.

To see that (30) provides a computable error estimator, introduce the basis in $V_{Q}=\tilde{V}$ in a hierarchical manner: take the nodal basis functions $N_{P}$ from $V_{L}=V$, and add the nodal basis functions $\tilde{N}_{e} \in V_{Q}$ corresponding to midpoints of edges into one set. Obviously, this basis of $\tilde{V}$ is well-conditioned in the following sense:

$$
b(\tilde{v}, \tilde{v}) \approx \sum_{P} c_{P}^{2}+\sum_{e} c_{e}^{2} \quad \forall \tilde{v}=\sum_{P} c_{P} N_{P}+\sum_{e} c_{e} \tilde{N}_{e} \in \tilde{V}
$$

Therefore,

$$
a(e, e) \approx b(\tilde{e}, \tilde{e}) \approx \sum_{P} b\left(\tilde{e}, N_{P}\right)^{2}+\sum_{e} b\left(\tilde{e}, \tilde{N}_{e}\right)^{2},
$$

where the moments are explicitly computable as

$$
b\left(\tilde{e}, N_{P}\right)=a\left(e, N_{P}\right)=0, \quad b\left(\tilde{e}, \tilde{N}_{e}\right)=a\left(e, \tilde{N}_{e}\right)=\tilde{\Phi}\left(\tilde{N}_{e}\right)-a\left(u_{L}, \tilde{N}_{e}\right)
$$

and do not require the knowledge of $u_{Q}$. Under the usual regularity assumptions, $a(e, e)$ is a reasonable substitute for the true error of the discrete solution $u_{L}$ in the energy norm and can be approximately computed via (31)-(32) by a sum of error indicators corresponding to the edges of the triangulation. Note that this error estimator is almost the same as used in the well-known adaptive code Kaskade [25]. It is tempting to develop this idea of deriving error estimators further. For similar work on this topic, see, for instance, [1, 2, 3, 31, 46].

\section{REFERENCES}

1. R. E. Bank, PLTMG - A software package for solving elliptic partial differential equations. User's guide 6.0, SIAM, Philadelphia, 1990. CMP 90:12

2. R. E. Bank, A. Weiser, Some a posteriori error estimators for elliptic partial differential equations, Math. Comp. 44 (1985), 283-301. MR 86g:65207

3. R. E. Bank, B. D. Welfert, A posteriori error estimates for the Stokes problem, SIAM J. Numer. Anal. 28 (1991), 591-623. MR 92a:65284 
4. F. A. Bornemann, H. Yserentant, A basic norm equivalence for the theory of multilevel methods, Numer. Math. 64 (1993), 455-476. MR 94b:65155

5. D. Braess, Finite Elemente, Springer, Berlin, 1992.

6. D. Braess, R. Verfürth, Multigrid methods for nonconforming finite element methods, SIAM J. Numer. Anal. 27 (1990), 979-986. MR 91j:65164

7. J. H. Bramble, Multigrid methods, Pitman Research Notes in Mathematical Sciences, vol. 294, Longman Sci.\&Techn., Harlow, Essex, 1993. MR 95b:65002

8. J. H. Bramble, J. E. Pasciak, J. Xu, The analysis of multigrid algorithms with nonnested spaces or noninherited quadratic forms, Math. Comp. 56 (1991), 1-34. MR 91h:65159

9. J. H. Bramble, J. E. Pasciak, J. Xu, Parallel multilevel preconditioners, Math. Comp. 55 (1990), 1-22. MR 90k:65170

10. S. Brenner, An optimal-order multigrid method for P1 nonconforming finite elements, Math. Comp. 52 (1989), 1-15. MR 89f:65119

11. S. Brenner, An optimal-order nonconforming multigrid method for the biharmonic equation, SIAM J.Numer. Anal. 26 (1989), 1124-1138. MR 90i:65189

12. S. Brenner, A nonconforming multigrid method for the stationary Stokes equations, Math. Comp. 55 (1990), 411-437. MR 91d:65167

13. S. Brenner, Multigrid methods for nonconforming finite elements, in Proc. Fourth Copper Mountain Conf. on Multigrid Methods (J. Mandel et al., eds.), SIAM, Philadelphia, 1989, pp. 54-65. MR 91h:65189

14. S. Brenner, A multigrid algorithm for the lowest-order Raviart-Thomas mixed triangular finite element method, SIAM J. Numer. Anal., 29 (1992), 647-678. MR 93j:65175

15. S. Brenner, Two-level additive Schwarz preconditioners for nonconforming finite element methods, preprint, Univ. of South Carolina, 1993.

16. S. Brenner, A two-level additive Schwarz preconditioner for nonconforming plate elements, preprint, Univ. of South Carolina, 1993.

17. S. Brenner, A two-level additive Schwarz preconditioner for macroelement approximations of the plate bending problem, preprint, Univ. of South Carolina, 1993.

18. F. Brezzi, M. Fortin, Mixed and hybrid finite element methods, Springer, Berlin, 1991. MR 92d:65187

19. P. G. Ciarlet, The finite element method for elliptic problems, North-Holland, Amsterdam, 1978. MR 58:25001

20. P. G. Ciarlet, Basic error estimates for elliptic problems, in Handbook of Numerical Analysis, vol. II: Finite element methods (part 1) (P. G. Ciarlet, J.-L. Lions, eds.), North Holland, Amsterdam, 1991, pp. 17-351. CMP 91:14

21. L. C. Cowsar, Domain decomposition methods for nonconforming finite element spaces of Lagrange type, in Proc. 6th Copper Mountain Conference on Multigrid Methods (N. D. Melson et al., eds.), NASA Conf. Publ. 3224, 1993, pp. 93-109.

22. M. Crouzeix, P. A. Raviart, Conforming and nonconforming finite element methods for solving the stationary Stokes equation, RAIRO Anal. Numér. 7, R-3 (1973), 33-76. MR 49:8401

23. W.Dahmen, A.Kunoth, Multilevel preconditioning, Numer. Math. 63 (1992), 315-344. MR 93j:65065

24. W. Dahmen, P. Oswald, X.-Q. Shi, $C^{1}$-hierarchical bases, J. Comp. Appl. Math. 51 (1994), 37-56. MR 95e:65098

25. P. Deuflhard, P. Leinen, H. Yserentant, Concepts of an adaptive finite element code, IMPACT, Comput. Sci. Engng. 1 (1989), 3-35.

26. W. Dörfler, Hierarchical bases for elliptic problems, Math.Comp. 58 (1992), 513-529. MR 92f: 65134

27. W. Dörfler, A note on the preconditioner by Bramble, Pasciak, and Xu, preprint, Inst. für Angew. Math., Univ. Zürich, 1991.

28. V. Girault, P. A. Raviart, Finite element methods for Navier-Stokes equations, Springer, Berlin, 1986. MR 88b:65129

29. M. Griebel, P. Oswald, On the abstract theory of additive and multiplicative Schwarz algorithms, Numer. Math. 70 (1995),163-180. CMP 95:10

30. M. R. Hanisch, Multigrid preconditioning for the biharmonic Dirichlet problem, SIAM J. Numer. Anal. 30 (1993), 184-214. MR 94e:65131

31. R. H. W. Hoppe, B. Wohlmuth, Element-oriented and edge-oriented error estimators for nonconforming finite element methods, preprint, TU Munich, 1992. 
32. G. A. Mohr, Finite elements for solids, fluids, and optimization, Oxford Univ. Press, Oxford, 1992. MR 95f:65003

33. S. V. Nepomnyaschikh, Decomposition and fictitious domain methods for elliptic boundary value problems, in 5th. Conf. on Domain Decomposition Methods for Partial Differential Equations (T. F. Chan et al., eds.), SIAM, Philadelphia, 1992, pp. 62-72. MR 93i:65116

34. S. V. Nepomnyaschikh, Fictitious components and subdomain alternating methods, Sov. J. Numer. Anal. Math. Modelling 5 (1990), 53-68.

35. P. Oswald, On a hierarchical basis multilevel method with nonconforming P1 elements, Numer. Math. 62 (1992), 189-212. MR 93b:65059

36. P. Oswald, On a BPX-preconditioner for P1 elements, Computing 51 (1993), 125-133. MR 94k:65064

37. P. Oswald, Hierarchical conforming finite element methods for the biharmonic equation, SIAM J. Numer. Anal. 29 (1992), 1610-1625. MR 93k:65098

38. P. Oswald, Multilevel finite element approximation: theory and applications, Teubner Skripten zur Numerik, Teubner, Stuttgart, 1994. CMP 95:06

39. P. Oswald, Multilevel preconditioners for discretizations of the biharmonic equation by rectangular finite elements, J. Numer. Lin. Alg. Appl. 2 (1995) (to appear).

40. P. Peisker, D. Braess, A conjugate gradient method and a multigrid algorithm for Morley's finite element approximation of the biharmonic equation, Numer. Math. 50 (1987), 567-586. MR 88e: 65147

41. P. Peisker, W. Rust, E. Stein, Iterative solution methods for plate bending problems: multigrid and preconditioned cg algorithms, SIAM J. Numer. Anal. 27 (1990), 1450-1465. MR 91h:73055

42. J. Pitkäranta, Analysis of some low-order finite element schemes for Reissner-Mindlin and Kirchhoff plates, Numer. Math. 53 (1988), 237-254. MR 89f:65126

43. M. Sarkis, Two-level Schwarz methods for nonconforming finite elements and discontinuous coefficients, in Proc. 6th Copper Mountain Conference on Multigrid Methods (N. D. Melson et al., eds.), NASA Conf. Publ. 3224, 1993, pp. 543-565.

44. P. Trouve, Three basic nonconforming approximations of thin plates and shells: the semiloof, the delinquent, and the DKT finite element methods, in The Mathematics of Finite Elements and Applications VII MAFELAP 1990 (J. R. Whiteman, ed.), Acad. Press, London, 1991, pp. 459-469. CMP 92:03

45. P. S. Vassilevski, J. Wang, An application of the abstract multilevel theory to nonconforming finite element methods, SIAM J. Numer. Anal. 32 (1995), 235-248. CMP 95:07

46. R. Verfürth, A posteriori error estimators for the Stokes equations, Numer. Math. 55 (1989), 309-325. MR 90d:65187

47. J. Xu, Iterative methods by space decomposition and subspace correction, SIAM Review 34 (1992), 581-613. MR 93k:65029

48. J. Xu, Convergence estimates for some multigrid algorithms, in III. Int. Symp. on Domain Decomposition Methods for Partial Differential Equations (T. F. Chan, R. Glowinski, J. Periaux, O. B. Widlund, eds.), SIAM, Philadelphia, 1990, pp. 174-187. MR 91f:65194

49. H. Yserentant, Old and new convergence proofs for multigrid methods, Acta Numerica, 1993, Cambr. Univ. Press, Cambridge, 1993, pp. 285-326. MR 94i:65128

50. X. Zhang, Multilevel Schwarz methods for the biharmonic Dirichlet problem, SIAM J. Sci. Comput. 15 (1994), 621-644. CMP 94:11

Institut für Angewandte Mathematik, FSU Jena, D-07740 Jena, Germany

Current address: Department of Mathematics, Texas A\&M University, College Station, Texas 77843

E-mail address: peter.oswald@math.tamu.edu 